\title{
An intrinsic metric for power spectral density functions*
}

\author{
Tryphon T. Georgiou ${ }^{\dagger}$
}

\begin{abstract}
We present an intrinsic metric that quantifies distances between power spectral density functions. The metric was derived by the author in [3] as the geodesic distance between spectral density functions with respect to a particular pseudo-Riemannian metric motivated by a quadratic prediction problem. We provide an independent verification of the metric inequality and discuss certain key properties of the induced topology.
\end{abstract}

\section{Index Terms}

Power spectral density functions, intrinsic metric, information geometry.

\section{THE METRIC PROPERTY}

7 HE present work builds on a recent report [3] where the present author introduced a natural pseudo-Riemannian metric on power spectral density functions of discrete-time stochastic processes, characterized geodesics, and computed geodesic distances. The geodesic distance between two power spectral density functions $f_{i}(\theta)$, with $i=1,2$ and $\theta \in[-\pi, \pi]$, was shown to be

$$
d_{g}\left(f_{1}, f_{2}\right):=\sqrt{\int_{-\pi}^{\pi}\left(\log \frac{f_{1}(\theta)}{f_{2}(\theta)}\right)^{2} \frac{d \theta}{2 \pi}-\left(\int_{-\pi}^{\pi} \log \frac{f_{1}(\theta)}{f_{2}(\theta)} \frac{d \theta}{2 \pi}\right)^{2}} .
$$

Below we will provide a direct verification that $d_{g}(\cdot, \cdot)$ provides a pseudo-metric on the cone of power spectral density functions

$$
\mathcal{D}:=\left\{f: f(\theta) \geq 0 \text { for } \theta \in[-\pi, \pi], f \in L_{1}[-\pi, \pi]\right\} .
$$

(As usual, $L_{1}, L_{2}$ denote Lebeague spaces of integrable and square-integrable functions, respectively.)

The only reason $d_{g}(\cdot, \cdot)$ is a pseudo-metric and not a metric is because it is insensitive to scaling, i.e., $d_{g}\left(f_{1}, f_{2}\right)=d_{g}\left(f_{1}, \kappa f_{2}\right)$ for any $\kappa>0$. Thus, it does not differentiate between spectral densities which only differ by a constant nonzero positive factor. Families of spectral density functions related in this way are referred to as spectral rays and form a set

$$
\mathcal{R}:=\left\{f \bmod \mathbb{R}_{+}: f(\theta) \geq 0 \text { for } \theta \in[-\pi, \pi], f \in L_{1}[-\pi, \pi]\right\}
$$

\footnotetext{
${ }^{\dagger}$ Department of Electrical and Computer Engineering, University of Minnesota, Minneapolis, MN 55455; tryphon@umn.edu. ${ }^{*}$ This research has been supported by the NSF and the AFOSR.
} 
of equivalence classes, and $d_{g}(\cdot, \cdot)$ can be used to evaluate distances on $\mathcal{R}$ via comparing any two representatives on any two given spectral rays. Then, as we will see, $d_{g}(\cdot, \cdot)$ defines a metric on $\mathcal{R}$. This metric can be also be readily modified to provide a metric on $\mathcal{D}$ if for instance, $\left|\int_{-\pi}^{\pi}\left(f_{1}(\theta)-f_{2}(\theta)\right) \frac{d \theta}{2 \pi}\right|$, or the absolute difference of any other generalized means is added on as in

$$
d\left(f_{1}, f_{2}\right):=d_{g}\left(f_{1}, f_{2}\right)+\left|\int_{-\pi}^{\pi}\left(f_{1}(\theta)-f_{2}(\theta)\right) \frac{d \theta}{2 \pi}\right|
$$

to differentiate the effect of scaling.

Before we proceed, we clarify how to evaluate $d_{g}(\cdot, \cdot)$ on all spectra in $\mathcal{D}$, including those that may vanish on a subset of the frequency interval $[-\pi, \pi]$ rendering $\log \left(f_{1} / f_{2}\right)^{2}$ non-integrable. Clearly, when neither argument of $d_{g}\left(f_{1}, f_{2}\right)$ vanishes and $f_{i}(\theta)$ stays away from zero for $\theta \in[-\pi, \pi]$ and $i=1,2$, then $\log f_{i} \in L_{2}[-\pi, \pi]$ and $d_{g}\left(f_{1}, f_{2}\right)$ is well defined and finite. But, if either $f_{i}(i=1,2)$ vanishes on $[-\pi, \pi]$ the integrals may diverge. However, since the root-mean-square of any function, and hence of $\log \left(f_{1} / f_{2}\right)$ in particular, is always greater than or equal to its arithmetic mean (e.g., see [2]) it follows that

$$
\sqrt{\int_{-\pi}^{\pi}\left(\log \frac{f_{1}(\theta)}{f_{2}(\theta)}\right)^{2} \frac{d \theta}{2 \pi}} \geq \int_{-\pi}^{\pi} \log \frac{f_{1}(\theta)}{f_{2}(\theta)} \frac{d \theta}{2 \pi}
$$

Therefore, (11) gives either a nonnegative real or has to be assigned the value $+\infty$. In conclusion, we complete the definition of $d_{g}(\cdot, \cdot)$ as follows: if

$$
\log \frac{f_{1}}{f_{2}} \in L_{2}[-\pi, \pi]
$$

in which case the left hand side of (2) is finite, $d_{g}\left(f_{1}, f_{2}\right)$ is evaluated using (1). If however (3) fails then, for consistency with (1), we assign

$$
d_{g}\left(f_{1}, f_{2}\right):=\infty
$$

Clearly, failure of (3) can always be traced to at least one of $f_{i}(i \in\{1,2\})$ failing to satisfy $\log f_{i} \in$ $L_{2}[-\pi, \pi]$ (otherwise, necessarily, $\left.\log \left(f_{1} / f_{2}\right)=\left(\log f_{1}-\log f_{2}\right) \in L_{2}[-\pi, \pi]\right)$.

Theorem 1: $d_{g}(\cdot, \cdot)$ defines a pseudo-metric on $\mathcal{D}$ and a metric on $\mathcal{R}$.

Proof: By definition $d(\cdot, \cdot) \in[0, \infty) \cup\{+\infty\}$. It is also easy to observe that

$$
d_{g}\left(f_{1}, f_{2}\right)=d_{g}\left(f_{2}, f_{1}\right)
$$

To see this note that $\log \left(f_{1} / f_{2}\right)=-\log \left(f_{2} / f_{1}\right)$ and that (1) is impervious to a sign change in front of the logarithms. Also in case one of $\log \left(f_{1} / f_{2}\right), \log \left(f_{2} / f_{1}\right)$ fails to be in $L_{2}$, so does the other, and again $d_{g}\left(f_{1}, f_{2}\right)=d_{g}\left(f_{2}, f_{1}\right)$ (both being $\infty$ ). Thus, (5) holds. 
When $d_{g}\left(f_{1}, f_{2}\right)=0$ the root-mean-square of the function $\log \left(f_{1} / f_{2}\right)$ is equal to its arithmetic mean, and this only happens (see [2]) when the function is constant, i.e.,

$$
\begin{aligned}
d_{g}\left(f_{1}, f_{2}\right)=0 & \Rightarrow \log \frac{f_{1}(\theta)}{f_{2}(\theta)}=c \in \mathbb{R} \text { for all } \theta \in[-\pi, \pi] \\
& \Rightarrow f_{1}=\kappa f_{2} \\
& \Rightarrow f_{1} \bmod \mathbb{R}=f_{2} \bmod \mathbb{R},
\end{aligned}
$$

since $\kappa=e^{c}$ is a constant. Thus, $d_{g}(\cdot, \cdot)$ separates the elements of $\mathcal{R}$.

We finally establish the triangular inequality. So let us consider $f_{i} \in \mathcal{D}$ for $i \in\{1,2,3\}$. We will show that

$$
d_{g}\left(f_{1}, f_{2}\right)+d_{g}\left(f_{2}, f_{3}\right) \geq d_{g}\left(f_{1}, f_{3}\right)
$$

We first argue the case when $d_{g}\left(f_{1}, f_{3}\right)=\infty$. It suffices to show that one of the left hand side terms is also infinity. Assume the contrary, i.e., that

$$
\log \frac{f_{1}}{f_{2}} \in L_{2}[-\pi, \pi] \text { as well } \log \frac{f_{2}}{f_{3}} \in L_{2}[-\pi, \pi] .
$$

It readily follows that $\log \left(f_{1} / f_{3}\right)=\log \left(f_{1} / f_{2}\right)+\log \left(f_{2} / f_{3}\right) \in L_{2}[-\pi, \pi]$ which contradicts the assumption that $d_{g}\left(f_{1}, f_{3}\right)=\infty$. Thus, at least one of $d_{g}\left(f_{1}, f_{2}\right), d_{g}\left(f_{2}, f_{3}\right)$ is infinity and the triangular inequality holds. Of course, if $\log \left(f_{1} / f_{3}\right)$ is finite and any of $d_{g}\left(f_{1}, f_{2}\right), d_{g}\left(f_{2}, f_{3}\right)$ takes the value $\infty$, the triangular inequality holds anyway.

We now argue the case when all three $d_{g}\left(f_{1}, f_{2}\right), d_{g}\left(f_{2}, f_{3}\right)$ and $d_{g}\left(f_{1}, f_{3}\right)$ are finite. To this end we square both sides of (6) and utilize

$$
\log \frac{f_{1}}{f_{3}}=\log \frac{f_{1}}{f_{2}}+\log \frac{f_{2}}{f_{3}},
$$

to simplify the resulting expression and deduce the following inequality

$$
\begin{aligned}
& \sqrt{\int_{-\pi}^{\pi}\left(\log \frac{f_{1}}{f_{2}}\right)^{2} \frac{d \theta}{2 \pi}-\left(\int_{-\pi}^{\pi} \log \frac{f_{1}}{f_{2}} \frac{d \theta}{2 \pi}\right)^{2}} \sqrt{\int_{-\pi}^{\pi}\left(\log \frac{f_{2}}{f_{3}}\right)^{2} \frac{d \theta}{2 \pi}-\left(\int_{-\pi}^{\pi} \log \frac{f_{2}}{f_{3}} \frac{d \theta}{2 \pi}\right)^{2}} \\
\geq & \int_{-\pi}^{\pi}\left(\log \frac{f_{1}}{f_{2}} \log \frac{f_{2}}{f_{3}}\right) \frac{d \theta}{2 \pi}-\left(\int \log \frac{f_{1}}{f_{2}} \frac{d \theta}{2 \pi}\right)\left(\int \log \frac{f_{2}}{f_{3}} \frac{d \theta}{2 \pi}\right) .
\end{aligned}
$$

Thus the two inequalities (8) and (6) are equivalent to one another, and therefore, in order to ascertain (6) it suffices to establish the validity of (8).

To this end, let $\alpha:=\log \left(f_{1} / f_{2}\right), \beta:=\log \left(f_{2} / f_{3}\right)$ and rewrite 8 in the form

$$
\begin{aligned}
& \sqrt{\int_{-\pi}^{\pi} \alpha^{2} \frac{d \theta}{2 \pi}-\left(\int_{-\pi}^{\pi} \alpha \frac{d \theta}{2 \pi}\right)^{2}} \sqrt{\int_{-\pi}^{\pi} \beta^{2} \frac{d \theta}{2 \pi}-\left(\int_{-\pi}^{\pi} \beta \frac{d \theta}{2 \pi}\right)^{2}} \\
\geq & \int_{-\pi}^{\pi}(\alpha \beta) \frac{d \theta}{2 \pi}-\left(\int_{-\pi}^{\pi} \alpha \frac{d \theta}{2 \pi}\right)\left(\int_{-\pi}^{\pi} \beta \frac{d \theta}{2 \pi}\right) .
\end{aligned}
$$


Since (9) is homogeneous in both $\alpha$ and $\beta$, scaling of either leaves it unaffected. Therefore, if

$$
\begin{aligned}
\sigma_{\alpha} & =\sqrt{\int_{-\pi}^{\pi} \alpha^{2} \frac{d \theta}{2 \pi}-\left(\int_{-\pi}^{\pi} \alpha \frac{d \theta}{2 \pi}\right)^{2}} \\
\sigma_{\beta} & =\sqrt{\int_{-\pi}^{\pi} \beta^{2} \frac{d \theta}{2 \pi}-\left(\int_{-\pi}^{\pi} \beta \frac{d \theta}{2 \pi}\right)^{2}}
\end{aligned}
$$

and $a:=\frac{1}{\sigma_{\alpha}} \alpha, b:=\frac{1}{\sigma_{\beta}} \beta$, the inequality (9) is equivalent to

$$
1 \geq \int_{-\pi}^{\pi}(a b) \frac{d \theta}{2 \pi}-\left(\int_{-\pi}^{\pi} a \frac{d \theta}{2 \pi}\right)\left(\int_{-\pi}^{\pi} b \frac{d \theta}{2 \pi}\right)
$$

with the side conditions

$$
\begin{aligned}
& \int_{-\pi}^{\pi} a^{2} \frac{d \theta}{2 \pi}-\left(\int_{-\pi}^{\pi} a \frac{d \theta}{2 \pi}\right)^{2}=1, \text { and } \\
& \int_{-\pi}^{\pi} b^{2} \frac{d \theta}{2 \pi}-\left(\int_{-\pi}^{\pi} b \frac{d \theta}{2 \pi}\right)^{2}=1 .
\end{aligned}
$$

But the validity of (10) follows trivially from the standard inequality

$$
\int_{-\pi}^{\pi}(a-b)^{2} \frac{d \theta}{2 \pi} \geq\left(\int_{-\pi}^{\pi}(a-b) \frac{d \theta}{2 \pi}\right)^{2}
$$

after we expand the squares on both sides and use (11) and (12) to simplify the resulting expressions. Thus (10) with (11, 12) holds $\Rightarrow(9) \Rightarrow(6)$, and this completes the proof.

Remark: The definition of $d_{g}(\cdot, \cdot)$ distinguishes two classes of power spectral densities according to whether their logarithm is square integrable or not. The first class, $\mathcal{D}_{\text {interior }}:=\{f \in \mathcal{D}: \log f \in$ $\left.L_{2}[-\pi, \pi]\right\}$, can be thought of as "interior" points lying to within a finite distance from one another, and to within a finite distance from constant non-zero power spectral densities. The second class, with logarithms that fail to be square integrable, contains power spectral densities which lie at an infinite distance from any density in $\mathcal{D}_{\text {interior. }}$ On the other hand, power spectal densities are traditionally differentiated according to whether the underlying process is deterministic or not. More specifically, a stochastic process is said to be non-deterministic (in the sense of Kolmogoroff) if the variance of the one-step-ahead prediction error cannot be made arbitrarily small. In turn, this property is characterized by the log-integrability of the corresponding power spectral density function (see [4], [5]), i.e., $\mathcal{D}_{\text {non-deterministic }}:=\{f \in \mathcal{D}:$ $\left.\log f \in L_{1}[-\pi, \pi]\right\}$. Thus, it is interesting to observe that $\mathcal{D}_{\text {interior }} \subset \mathcal{D}_{\text {non-deterministic }}$ and hence, finite neighborhoods of elements in $\mathcal{D}_{\text {interior }}$ contain non-deterministic power spectra only.

\section{RIEMANNIAN GEOMETRY, GEODESICS, AND INTRINSIC METRICS}

We now explain the geometric significance of $d_{g}(\cdot, \cdot)$ recapitulating some of the development in [3]. The starting point that led to (11) is a prediction problem and the degradation of the variance of the prediction error when the design of the predictor is based on the wrong choice among two alternatives. More 
specifically, let $f_{1}, f_{2}$ represent spectral density functions of discrete-time zero-mean stochastic processes $u_{f_{i}}(k)(i \in\{1,2\}$ and $k \in \mathbb{Z})$, and let $p_{f_{i}}(\ell)(\ell \in\{1,2,3, \ldots\})$ represent values for the coefficients that minimize the linear prediction error variance

$$
\mathcal{E}\left\{\left|u_{f_{i}}(0)-\sum_{\ell=1}^{\infty} p(\ell) u_{f_{i}}(-\ell)\right|^{2}\right\} .
$$

Thus, the optimal set of coefficients depends on the power spectral density function of the process, a fact which is duly acknowledged by the subscript in the notation $p_{f_{i}}(\ell)$. Here, as usual, $\mathcal{E}\{\}$ denotes the expectation operator. It is reasonable to consider as a distance between $f_{1}$ and $f_{2}$ the degradation of predictive error variance when the coefficients $p(\ell)$ are selected assuming one of the two, and then used to predict a stochastic process corresponding to the other spectral density function. The ratio of the "degraded" predictive error variance over the optimal error variance

$$
\rho\left(f_{1}, f_{2}\right):=\frac{\mathcal{E}\left\{u_{f_{1}}(0)-\left.\sum_{\ell=1}^{\infty} p_{f_{2}}(\ell) u_{f_{1}}(-\ell)\right|^{2}\right\}}{\mathcal{E}\left\{u_{f_{1}}(0)-\left.\sum_{\ell=1}^{\infty} p_{f_{1}}(\ell) u_{f_{1}}(-\ell)\right|^{2}\right\}}
$$

turns out to be equal to the ratio of the arithmetic over the geometric means of the fraction of the two spectral density functions, namely

$$
\rho\left(f_{1}, f_{2}\right)=\frac{\int_{-\pi}^{\pi} \frac{f_{1}(\theta)}{f_{2}(\theta)} \frac{d \theta}{2 \pi}}{\exp \left(\int_{-\pi}^{\pi} \log \left(\frac{f_{1}(\theta)}{f_{2}(\theta)}\right) \frac{d \theta}{2 \pi}\right)},
$$

see [3].

The logarithm $\log \rho\left(f_{1}, f_{2}\right)=: \delta_{\mathrm{a} / \mathrm{g}}\left(f_{1}, f_{2}\right)$ (where the subscript a/g signifies arithmetic/geometric) represents a measure of dissimilarity between the "shapes" of $f_{1}$ and $f_{2}$ and, can be viewed, as analogous to "divergences" of Information Theory. Indeed,

$$
\delta_{\mathrm{a} / \mathrm{g}}\left(f_{1}, f_{2}\right)=\log \left(\int_{-\pi}^{\pi} \frac{f_{1}(\theta)}{f_{2}(\theta)} \frac{d \theta}{2 \pi}\right)-\int_{-\pi}^{\pi} \log \left(\frac{f_{1}(\theta)}{f_{2}(\theta)}\right) \frac{d \theta}{2 \pi}
$$

vanishes only when $f_{1} / f_{2}$ is constant on $[-\pi, \pi]$ and is positive otherwise. Considering the distance $\delta_{\mathrm{a} / \mathrm{g}}(f, f+\Delta)$ between a nominal power spectral density $f$ and a perturbations $f+\Delta$, and eliminating cubic terms and beyond, leads (modulo a scaling factor of 2) to the Riemannian pseudo-metric in $\mathcal{D}_{\text {interior }}$ which is given by the following quadratic differential form

$$
g_{f}(\Delta):=\int_{-\pi}^{\pi}\left(\frac{\Delta(\theta)}{f(\theta)}\right)^{2} \frac{d \theta}{2 \pi}-\left(\int_{-\pi}^{\pi} \frac{\Delta(\theta)}{f(\theta)} \frac{d \theta}{2 \pi}\right)^{2} .
$$

Interestingly, geodesic paths $f_{\tau}(\tau \in[0,1])$ connecting spectral densities $f_{0}, f_{1}$ and having minimal length

$$
\sqrt{2} \int_{0}^{1} \sqrt{\delta_{\mathrm{a} / \mathrm{g}}\left(f_{\tau}, f_{\tau+d \tau}\right)}=\int_{0}^{1} \sqrt{g_{f_{\tau}}\left(\frac{\partial f_{\tau}}{\partial \tau}\right)} d \tau
$$

can be explicitely computed [3]. They turn out to be logarithmic intervals

$$
f_{\tau}(\theta)=f_{0}^{1-\tau}(\theta) f_{1}^{\tau}(\theta) \text { for } \tau \in[0,1]
$$


between the two extreme points. Furthermore, the length along such geodesics is precisely $d_{g}\left(f_{0}, f_{1}\right)$ as given in (1).

The closed form of the geodesic path allows us to verify directly that any two power spectral densities $f_{0}, f_{1}$, at a finite distance from one another, can be connected with a path of the same length. A topological space with such a property is said to be a length-space and the metric is said to be intrinsic. The fact that $d_{g}(\cdot, \cdot)$ is intrinsic can be readily verified and this is done below.

Proposition 2: $d_{g}(\cdot, \cdot)$ is intrinsic on $\mathcal{D}$ and $\mathcal{R}$.

Proof: By direct substitution into (11) we can verify that for any $f_{0}, f_{1}$ such that $d_{g}\left(f_{0}, f_{1}\right)<\infty$, any $\tau \in[0,1]$, and with $f_{\tau}$ defined as in (14), $d_{g}\left(f_{0}, f_{\tau}\right)=\tau d_{g}\left(f_{0}, f_{1}\right), d_{g}\left(f_{\tau}, f_{1}\right)=(1-\tau) d_{g}\left(f_{0}, f_{1}\right)$, and even $d_{g}\left(f_{\tau}, f_{\tau+d \tau}\right)=d_{g}\left(f_{0}, f_{1}\right) d \tau$. It readily follows that the length of the path $\int_{0}^{1} d_{g}\left(f_{\tau}, f_{\tau+d \tau}\right)=$ $\int_{0}^{1} d_{g}\left(f_{0}, f_{1}\right) d \tau$ equals the distance $d_{g}\left(f_{0}, f_{1}\right)$ between the end points.

Remark: Besides $\delta_{\mathrm{a} / \mathrm{g}}\left(f_{1}, f_{2}\right)$, several other "divergences" have been introduced in [3] as appropriate distance measures (though not metrics). First the symmetrized version

$$
\delta\left(f_{1}, f_{2}\right)=\delta_{\mathrm{a} / \mathrm{g}}\left(f_{1}, f_{2}\right)+\delta_{\mathrm{a} / \mathrm{g}}\left(f_{2}, f_{1}\right)
$$

and then,

$$
\delta_{r, s}\left(f_{1}, f_{2}\right):=\log \sqrt[r]{\int_{-\pi}^{\pi}\left(\frac{f_{1}}{f_{2}}\right)^{r} \frac{d \theta}{2 \pi}}-\log \sqrt[s]{\int_{-\pi}^{\pi}\left(\frac{f_{1}}{f_{2}}\right)^{s} \frac{d \theta}{2 \pi}}
$$

involving comparison of other generalized means. It is interesting to point out that the quadratic terms of $\delta_{r, s}(f, f+\Delta), \delta_{\mathrm{a} / \mathrm{g}}(f, f+\Delta)$ and $\delta(f, f+\Delta)$ in the "perturbation" $\Delta$, all turn out to be identical (modulo a scaling). Hence, they all lead to the same Riemannian pseudo-metric (13).

\section{CONCluding Thoughts}

It is interesting to compare the differential structure on power spectral density functions which we have introduced above with the corresponding differential structure of "Information Geometry." In Information Geometry $f(\theta)$ corresponds to a probability density on $[-\pi, \pi]$ and the natural Riemannian metric is the Fisher information metric is (cf. [1, page 28]) which is this case would be

$$
\begin{aligned}
g_{\text {Fisher }, f}(\Delta) & =\int_{-\pi}^{\pi}\left(\frac{\Delta(\theta)}{f(\theta)}\right)^{2} f(\theta) \frac{d \theta}{2 \pi} \\
& =\int_{-\pi}^{\pi} \frac{\Delta(\theta)^{2}}{f(\theta)} \frac{d \theta}{2 \pi}
\end{aligned}
$$

(with $\int_{-\pi}^{\pi} f(\theta) \frac{d \theta}{2 \pi}=1$ and $\int_{-\pi}^{\pi} \Delta(\theta) \frac{d \theta}{2 \pi}=0$ since both $f, f+\Delta$ need to be probability densities). Direct comparison reveals that the powers of $f(\theta)$ in (13) and (15) are different. Thus, it is curious and worth underscoring that in either differential structure, geodesics and geodesic lengths can be computed. 


\section{REFERENCES}

[1] S. Amari and H. Nagaoka, Methods of Information Geometry, Translations of Mathematical Monographs, AMS, Oxford University Press, 2000.

[2] E.F. Beckenbach and R. Bellman, Inequalities, Springer-Verlag, Berlin-Heidelberg, 198 pages,1965.

[3] T.T. Georgiou, "Distances between power spectral densities," submitted to the IEEE Trans. on Signal Processing, arXiv:math.OC/0607026 July 2006.

[4] U. Grenander and G. Szegö, Toeplitz Forms and their Applications, Chelsea, 1958.

[5] P. Stoica and R. Moses, Introduction to Spectral Analysis, Prentice Hall, 2005. 